\title{
Factors Affecting Canagliflozin-Induced Transient Urine Volume Increase in Patients with Type 2 Diabetes Mellitus
}

\author{
Hiroyuki Tanaka · Kazuhiko Takano · Hiroaki Iijima • \\ Hajime Kubo · Nobuko Maruyama - Toshio Hashimoto • Kenji Arakawa • \\ Masanori Togo · Nobuya Inagaki · Kohei Kaku
}

Received: October 6, 2016 / Published online: December 15, 2016

(C) The Author(s) 2016. This article is published with open access at Springerlink.com

\begin{abstract}
Introduction: Sodium glucose co-transporter 2 (SGLT2) inhibitors exhibit diuretic activity, which is a possible mechanism underlying the cardiovascular benefit of these inhibitors. However, the osmotic diuresis-induced

increase in urine volume, and the risk of dehydration have been of concern with SGLT2 inhibitor treatment. This study aimed to investigate the mechanism underlying SGLT2 inhibitor canagliflozin-induced diuresis in Japanese type 2 diabetes mellitus (T2DM) patients.
\end{abstract}

Enhanced content To view enhanced content for this article go to www.medengine.com/Redeem/A137F0600 6C04F1C.

Electronic supplementary material The online version of this article (doi:10.1007/s12325-016-0457-8) contains supplementary material, which is available to authorized users.

H. Tanaka

Medical Affairs Department II, Mitsubishi Tanabe

Pharma Corporation, Tokyo, Japan

K. Takano

Medical Corporation Hokubukai Utsukushigaoka

Hospital, Sapporo, Japan

H. Iijima $(\bowtie)$

Science Communication Department, Mitsubishi

Tanabe Pharma Corporation, Tokyo, Japan

e-mail: Iijima.Hiroaki@mm.mt-pharma.co.jp

H. Kubo

Ikuyaku Medical Research Department, Mitsubishi

Tanabe Pharma Corporation, Osaka, Japan

N. Maruyama

Clinical Planning Department II, Mitsubishi Tanabe

Pharma Corporation, Tokyo, Japan
Methods: Thirteen T2DM patients received a daily oral dose of $100 \mathrm{mg}$ canagliflozin before breakfast for 6 days. Blood and urine samples were collected at predetermined time points. The primary endpoint was evaluation of correlations between changes from baseline in

T. Hashimoto

Product Coordination Department, Mitsubishi

Tanabe Pharma Corporation, Tokyo, Japan

\section{K. Arakawa}

Ikuyaku Medical Research Department, Mitsubishi

Tanabe Pharma Corporation, Tokyo, Japan

M. Togo

Medical Science Center, Mitsubishi Tanabe Pharma Corporation, Osaka, Japan

\section{N. Inagaki}

Department of Diabetes, Endocrinology and Nutrition, Kyoto University Graduate School of Medicine, Kyoto, Japan

K. Kaku

Department of Internal Medicine, Kawasaki Medical School, Okayama, Japan 
urine volume and factors that are known to affect urine volume and between actual urine volume and these factors.

Results: Canagliflozin transiently increased urine volume and urinary sodium excretion on Day 1 with a return to baseline levels thereafter. Canagliflozin administration increased urinary glucose excretion, which was sustained during repeated-dose administration. Plasma atrial natriuretic peptide (ANP) and N-terminal pro-b-type natriuretic peptide (NT-proBNP) levels decreased, while plasma renin activity increased. On Day 1 of treatment, changes in sodium and potassium excretion were closely correlated with changes in urine output. A post hoc multiple regression analysis showed changes in sodium excretion and water intake as factors that affected urine volume change at Day 1. Furthermore, relative to that at baseline, canagliflozin decreased blood glucose throughout the day and increased plasma total GLP-1 after breakfast.

Conclusion: Canagliflozin induced transient sodium excretion and did not induce water intake at Day 1; hence, natriuresis rather than glucose-induced osmotic diuresis may be a major factor involved in the canagliflozin-induced transient increase in urine output. In addition, canagliflozin decreased plasma ANP and NT-proBNP levels and increased plasma renin activity, which may be a compensatory mechanism for sodium retention, leading to subsequent urine output recovery.

Clinical trial registration: UMIN000019462.

Funding: Mitsubishi Tanabe Pharma Corporation.

Keywords: Canagliflozin; Diuresis; Natriuresis; Sodium glucose co-transporter 2; Type 2 diabetes mellitus

\section{INTRODUCTION}

Typical features of type 2 diabetes mellitus (T2DM) are insulin resistance of various organs and reduced glucose-stimulated insulin secretion [1]. In addition, the kidney plays a crucial role in regulating glucose levels by mediating the reabsorption of glucose filtered through glomeruli. Renal glucose reabsorption is mediated by sodium glucose co-transporter 2 (SGLT2) and SGLT1, with SGLT2 playing the major role because of its specific localization in the kidney and higher capacity than SGLT1. SGLT2 expression is increased in T2DM; hence, higher amounts of glucose are reabsorbed by the kidney, thereby contributing to the sustained elevation of serum glucose levels in patients with diabetes [2, 3]. SGLT2 inhibitors reduce blood glucose levels by suppressing this glucose reabsorption in an insulin-independent manner. Furthermore, SGLT2 inhibitors have demonstrated not only a hypoglycemic effect but also a body weight-reducing effect through calorie loss associated with glycosuria and a hypotensive effect [3-5]. In addition to control of blood glucose, control of body weight and blood pressure play important roles in preventing complications associated with T2DM [6]. Thus, a series of SGLT2 inhibitors have become new therapeutic options for T2DM [3-5, 7-9]. Several studies of SGLT2 inhibitors have been conducted to assess the cardiovascular outcomes [10], and it was reported that the SGLT2 inhibitor empagliflozin reduced heart failure hospitalization as well as cardiovascular and all-cause deaths in T2DM patients with high CVD risk (EMPA-REG OUTCOME trial) [11]. One possible mechanism underlying the cardiovascular benefit of empagliflozin is related to its osmotic diuretic effect by 
glycosuria [12, 13]. However, osmotic diuresis-induced increase in urine volume, loss of body fluid, and risk of dehydration have been of concern with SGLT2 inhibitor treatment $[14,15]$.

Canagliflozin is an SGLT2 inhibitor approved in North America, Europe, Latin America, and Asia-Pacific regions for treatment of T2DM [16-18]. We previously conducted a pharmacokinetic and pharmacodynamic study of canagliflozin in Japanese patients with T2DM, in which administration of canagliflozin at $25,100,200$, or $400 \mathrm{mg}$ once-daily increased 24 -h urine volume by $400-700 \mathrm{~mL}$ on Day 1, with a return to baseline from Day 2 onward. There was also an increase in urinary sodium ( $\mathrm{Na}$ ) excretion on Day 1 with a return to baseline thereafter, while water intake increased in the 400-mg group on Day 1, but did not change in the other dosage groups [19] (ESM Table 1). Although some previous studies reported that SGLT2 inhibitor-induced increases in urine volume were short-lived [20-22], the detailed nature of the transient effect remains unknown. For the proper clinical use of the SGLT2 inhibitor canagliflozin, it is important to confirm this transient increase in urine volume and elucidate the underlying mechanism. Therefore, the purpose of this study was to investigate the mechanism underlying canagliflozin-induced diuresis in Japanese T2DM patients by measuring factors that affect homeostasis of body fluid and mineral balance.

\section{METHODS}

\section{Subjects and Study Design}

This single-arm, exploratory study was carried out to investigate the factors involved in the canagliflozin-induced transient change in urine volume in Japanese T2DM patients by examining major endogenous factors that affect urine volume. Eligible subjects were men and women with stable T2DM with the following characteristics: age of $\geq 25$ and $<75$ years at the time of providing written consent; inadequate glycemic control, comprising $\quad \mathrm{HbA}_{1 \mathrm{c}} \geq 7 \% \quad(53.01 \mathrm{mmol} / \mathrm{mol})$ and $\leq 10 \% \quad(85.8 \mathrm{mmol} / \mathrm{mol}) ; \quad \mathrm{BMI} \geq 20$ and $\leq 35 \mathrm{~kg} / \mathrm{m}^{2}$; on unchanged single or dual oral antidiabetic drug therapy for $\geq 3$ months; systolic blood pressure of $\geq 95$ and $\leq 160 \mathrm{mmHg}$ and diastolic blood pressure of $\geq 50$ and $\leq 100 \mathrm{mmHg}$; and an estimated GFR (eGFR) of $\geq 60 \mathrm{~mL} / \mathrm{min} / 1.73 \mathrm{~m}^{2}$. The major exclusion criteria were as follows: no treatment for T2DM; history of type 1 diabetes mellitus or secondary diabetes; history of repeated severe hypoglycemia, or on therapy with insulin, glucagon-like peptide 1 (GLP-1) receptor agonist, SGLT2 inhibitor, or diuretic; history of serious diabetic complications requiring treatment; history or complication of kidney disease, nephrolithiasis, prostatitis, or complication of urinary tract infection; history of drug or food allergy; myocardial infarction, congestive heart failure, unstable angina pectoris, or cerebrovascular disorder (except for lacuna infarction) within 6 months prior to screening; history of transient ischemic attack or brain infarction with apparent neurological symptoms; complication of arteriosclerosis obliterans (stage III or IV of Fontaine classification); history of atrial fibrillation; or patients whom the investigator judged to be inappropriate for this study.

Eligible patients were hospitalized for 8 days for the study. From the third day (Day 1) to the eighth day (Day 6), each patient received an oral daily dose of canagliflozin $100 \mathrm{mg}$ at 8:00 am 
before breakfast and was asked to finish their breakfast within $20 \mathrm{~min}$. Blood and urine samples were collected at predetermined time points for laboratory tests and analyses of factors affecting urine volume. Efficacy was evaluated using data from Day 0 to Day 5, and safety was evaluated using data from day of admission to Day 6.

\section{Endpoints and Assessments}

The primary endpoints were correlations between changes from baseline in urine volume and factors known to affect urine volume, and between actual urine volume and these factors. The factors examined included water intake, urinary glucose excretion, urinary electrolyte excretion ( $\mathrm{Na}$ and K), urine osmolality, and plasma concentrations of atrial natriuretic peptide (ANP), N-terminal pro-b-type natriuretic peptide (NT-proBNP), vasopressin, aldosterone, and renin activity. The blood samples for measuring these plasma hormones and enzyme (ANP, NT-proBNP, vasopressin, aldosterone, and renin) were obtained from the recumbent position. The secondary endpoints were blood glucose over time, changes in total GLP-1 concentration after breakfast, and safety such as adverse events (AEs), laboratory tests, and 12-lead ECGs. The daily urine osmolality, AUCs of blood glucose, and AUCs of total GLP-1 were determined by post hoc analysis. Laboratory tests including biochemical and hematological examinations, measurements of hormone levels, immunological tests, and urinalysis were performed by the Sapporo Clinical Laboratory Inc. (Sapporo, Japan). The 12-lead ECGs were monitored at the Clinical Pharmacology Center, Medical Corporation
Hokubukai Utsukushigaoka Hospital (Sapporo, Japan).

\section{Ethics Approval and Consent to Participate}

The study was conducted in accordance with ethical principles that complied with the Declaration of Helsinki (revised October 2013), ethical guidelines for medical and health research involving human subjects, and conflict of interest management rules for clinical studies at the Medical Corporation Hokubukai Utsukushigaoka Hospital (Sapporo, Japan). The study protocol was approved by the Institutional Review Board at the Utsukushigaoka Hospital. All participants provided written informed consent before taking part in the study. Clinical trial registration: UMIN000019462.

\section{Statistical Analysis}

To analyze the primary endpoint, Spearman's correlation coefficients between changes from baseline in urine volume and factors known to affect urine volume, as well as between actual urine volume and each factor, were calculated, and a statistical test for absence of correlation was performed. Regarding continuous variables including urine volume, summary statistics (mean, SD, median, minimum, and maximum) for actual values and changes from baseline at each time point were calculated. For changes from baseline, 95\% confidence intervals (CIs) were also calculated. For each of the secondary endpoints, actual values and changes from baseline were obtained at each time point and the data were analyzed in the same manner. To identify factors affecting a transient increase in urine volume, a post hoc multiple regression analysis (stepwise variable 
selection method; both entry and retention criteria were set at $p=0.05$.) was performed with change in urine volume as the dependent variable and related factors as independent variables.

Statistical analyses of the present study were performed with SAS 9.3 (SAS Institute Inc., Cary, NC, USA) and Microsoft Excel 2010 (Microsoft Corporation, Redmond, WA, USA). Values of $p<0.05$ were considered statistically significant.

Data management and statistical analyses were carried out by IBEC Co. Ltd. (Osaka, Japan).

To evaluate the reproducibility of the present study, correlation and multiple regression analyses of a previous study [19] were performed by Takumi Information Technology (Tokyo, Japan) using SAS 9.4 (SAS Institute Inc.).

\section{RESULTS}

\section{Patient Disposition and Baseline Characteristics}

The baseline demographic and disease characteristics of the patients are summarized in Table 1. A total of 14 patients were enrolled, of whom 13 received the treatment and 12 (10 males and 2 females) completed the study. One female patient withdrew from the study because of AEs including anorexia, malaise, nausea, and vomiting.

\section{Effect on Urine Volume and Changes from Baseline}

Canagliflozin treatment showed a trend towards increased urine volume by $267.1 \mathrm{~mL}$ (95\% CI: -70.5-604.7 mL) on Day 1. Subsequently, the urine volume returned to baseline from Day 2 to Day 4, and showed another increase trend on Day 5. As the dropout patient had abnormal
Table 1 Demographic and clinical characteristics of the patients

\begin{tabular}{|c|c|c|}
\hline Total & $n$ & 13 \\
\hline \multicolumn{3}{|l|}{ Sex } \\
\hline Male & $n$ & 10 \\
\hline Female & $n$ & 3 \\
\hline Age (years) & Mean (SD) & $51.2(9.4)$ \\
\hline Body weight $(\mathrm{kg})$ & Mean (SD) & $73.72(13.8)$ \\
\hline BMI $\left(\mathrm{kg} / \mathrm{m}^{2}\right)$ & Mean (SD) & $26.70(4.26)$ \\
\hline $\begin{array}{l}\text { Duration of T2DM } \\
\text { (years) }\end{array}$ & Mean (SD) & $6.33(4.08)$ \\
\hline HbAlc (\%) & Mean (SD) & $8.06(0.73)$ \\
\hline Blood glucose $(\mathrm{mg} / \mathrm{dL})$ & Mean $(\mathrm{SD})$ & $157.6(29.4)$ \\
\hline 24-h urine volume $(\mathrm{mL})$ & Mean $(\mathrm{SD})$ & $2835.5(1356.6)$ \\
\hline $\begin{array}{l}\mathrm{eGFR}^{\mathrm{a}}(\mathrm{mL} / \mathrm{min} / \\
\left.1.73 \mathrm{~m}^{2}\right)\end{array}$ & Mean (SD) & $81.79(20.57)$ \\
\hline \multicolumn{3}{|l|}{ Oral antidiabetics } \\
\hline No & $n$ & 4 \\
\hline Yes & $n$ & 9 \\
\hline
\end{tabular}

eGFR estimated GFR, T2DM type 2 diabetes mellitus

${ }^{a}$ At screening visit

water intake and urine volume after hospitalization, we also performed the analysis without this patient as a reference, and found that canagliflozin treatment increased urine volume by $362.9 \mathrm{~mL}$ (95\% CI: 71.6-654.2 mL) on Day 1 (Table 2).

\section{Effect on Parameters that Reflect Urine Volume}

Canagliflozin administration increased urinary glucose excretion on Day 1, and this level was maintained thereafter (Fig. 1a). Water intake did not change appreciably until Day 4, and showed a trend towards an increase on Day 5 (Fig. 1b). Urinary $\mathrm{Na}$ excretion tended to 
Table 2 Effect of canagliflozin on urine volume ${ }^{a}$

\begin{tabular}{|c|c|c|c|c|c|c|}
\hline \multirow{3}{*}{$\begin{array}{l}\text { Time } \\
\text { point }\end{array}$} & \multirow[t]{3}{*}{$n$} & \multicolumn{5}{|c|}{ Urine volume $(\mathrm{mL})$} \\
\hline & & \multicolumn{2}{|l|}{ Actual values } & \multicolumn{2}{|c|}{ Changes from day 0} & \multirow[t]{2}{*}{$95 \% \mathrm{CI}$} \\
\hline & & Mean & SD & Mean & SD & \\
\hline Day 0 & $13(12)$ & $2835.5(2667.7)$ & $1356.6(1268.3)$ & & & \\
\hline Day 1 & $13(12)$ & $3102.5(3030.6)$ & $1281.0(1310.2)$ & $267.1(362.9)$ & $558.6(458.5)$ & $\begin{array}{l}-70.5 \text { to } 604.7 \\
\quad(71.6 \text { to } 654.2)\end{array}$ \\
\hline Day 2 & $13(12)$ & $2623.2(2617.8)$ & $888.4(927.6)$ & $-212.3(-49.8)$ & $994.2(839.0)$ & $\begin{array}{l}-813.1 \text { to } 388.5 \\
\quad(-582.9 \text { to } 483.2)\end{array}$ \\
\hline Day 3 & $13(12)$ & $2340.8(2397.8)$ & $1168.6(1201.6)$ & $-494.7(-269.9)$ & $1032.7(668.4)$ & $\begin{array}{l}-1118.7 \text { to } 129.3 \\
\quad(-694.6 \text { to } 154.8)\end{array}$ \\
\hline Day 4 & 12 & 2708.4 & 1051.8 & 40.8 & 521.8 & -290.8 to 372.3 \\
\hline Day 5 & 12 & 2920.9 & 979.2 & 253.3 & 529.0 & -82.8 to 589.3 \\
\hline
\end{tabular}

${ }^{a}$ Data in parentheses are from patients who completed the study

increase on Day 1, but returned to baseline thereafter (Fig. 1c). Urinary $\mathrm{K}$ excretion tended to decrease on Day 2 and thereafter (Fig. 1d). Urine osmolality showed an increase immediately after canagliflozin administration (Fig. 1e). Serum osmolality, blood urea nitrogen-to-serum creatinine ratio (BUN/CRE), and hematocrit (Ht) did not change during treatment (Fig. 1f-h).

\section{Effect on Plasma Hormone Levels/Enzyme Activity}

The plasma concentrations of hormones and enzyme activity related to body fluid control were measured at predetermined time points, and their AUCs over $24 \mathrm{~h}$ were calculated. Canagliflozin caused a reduction in NT-proBNP at $24 \mathrm{~h}$ after administration, a decrease in ANP from $4 \mathrm{~h}$, and an increase in renin activity at Day 5. Reduced AUCs of NT-proBNP and ANP were observed on Day 5 and Days 1 and 5, respectively, and that of renin activity increased on Day 5 (Fig. 2a-c; Table 3). The plasma concentrations of aldosterone and vasopressin were not appreciably affected by the treatment (Fig. 2d, e; Table 3).

\section{Correlation and Multiple Regression Analyses}

Spearman's correlation coefficients were calculated between changes from baseline in urine volume and each factor, and also between urine volume and each factor (Table 4). Change from baseline in urine volume was correlated with changes from baseline in urinary glucose excretion, urinary $\mathrm{Na}$ excretion, and urinary $\mathrm{K}$ excretion on Day 1, and negatively correlated with change from baseline in aldosterone $\mathrm{AUC}_{0-24 \mathrm{~h}}$ on Day 5. Actual value of urine volume was correlated with water intake (Days 0,1 , and 5) and urine glucose excretion (Days 0 and 1), and negatively correlated with urine osmolality (Days 0, 1, and 5; Table 4). A similar analysis was performed using data from a previous study [19], which found that change from baseline in urine volume was correlated with changes from baseline in urinary $\mathrm{Na}$ and $\mathrm{K}$ excretions on Day 1 and was not correlated with 

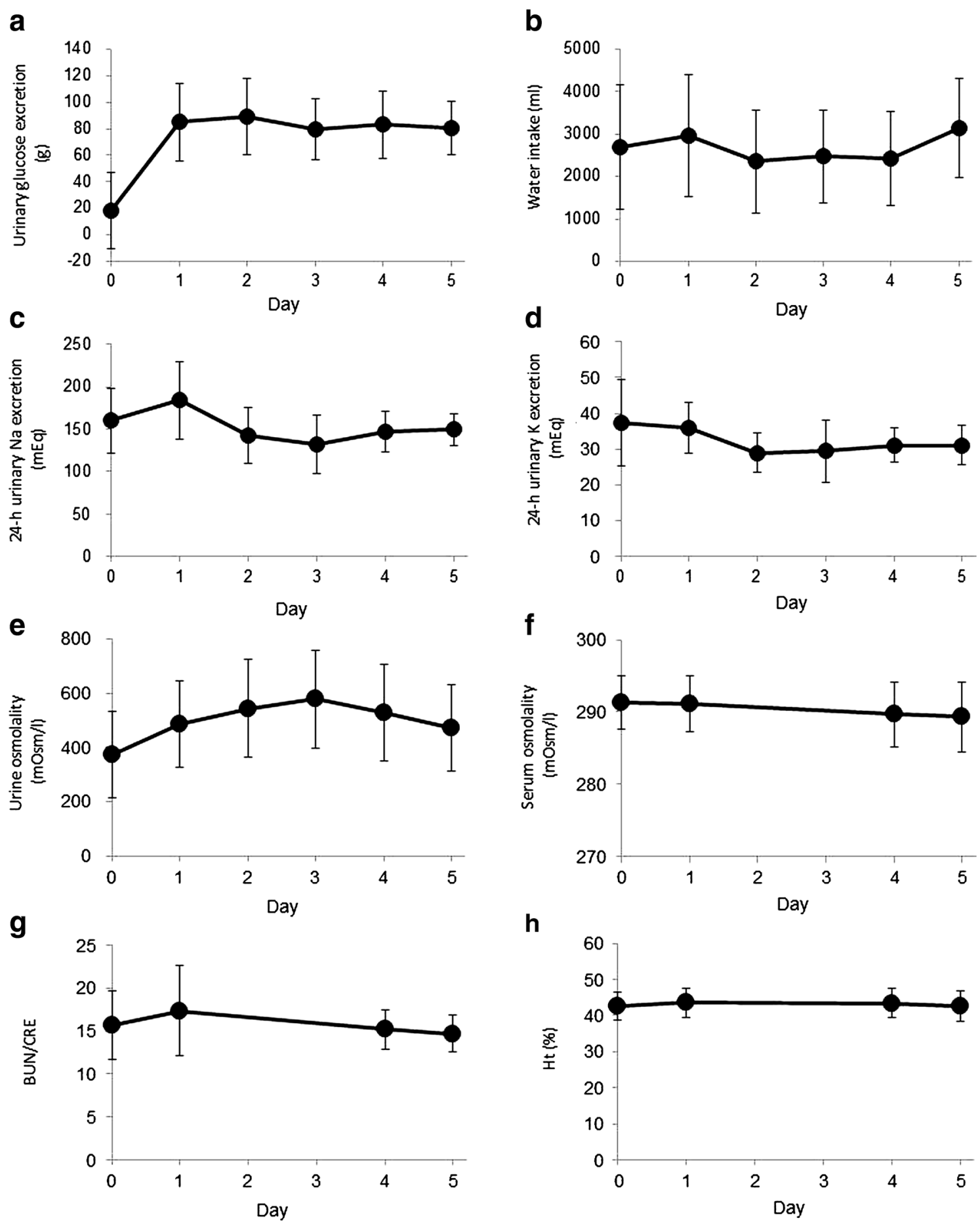

h

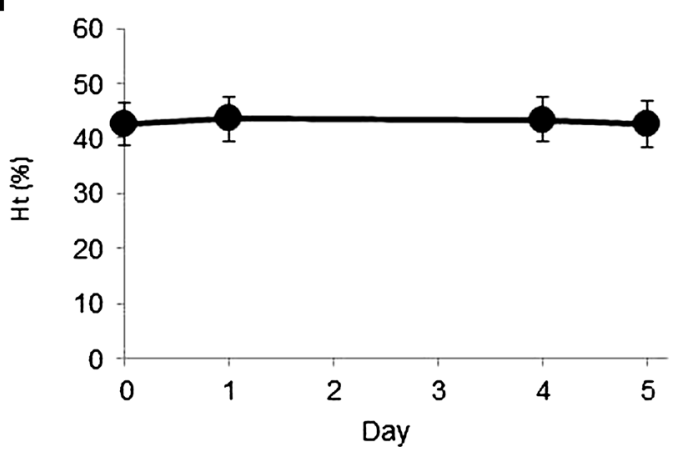

Fig. 1 Canagliflozin-induced changes in parameters that reflect urine volume. Changes in a 24-h urinary glucose excretion, b water intake, c 24-h urinary $\mathrm{Na}$ excretion, d 24-h urinary $\mathrm{K}$ excretion, e urine osmolality, f serum

osmolality, g BUN/CRE, and h Ht. Numbers of patients: days $0,1,2$, and $3, n=13$; days 4 and $5, n=12$. Data are expressed as mean $\pm \mathrm{SD}$ 

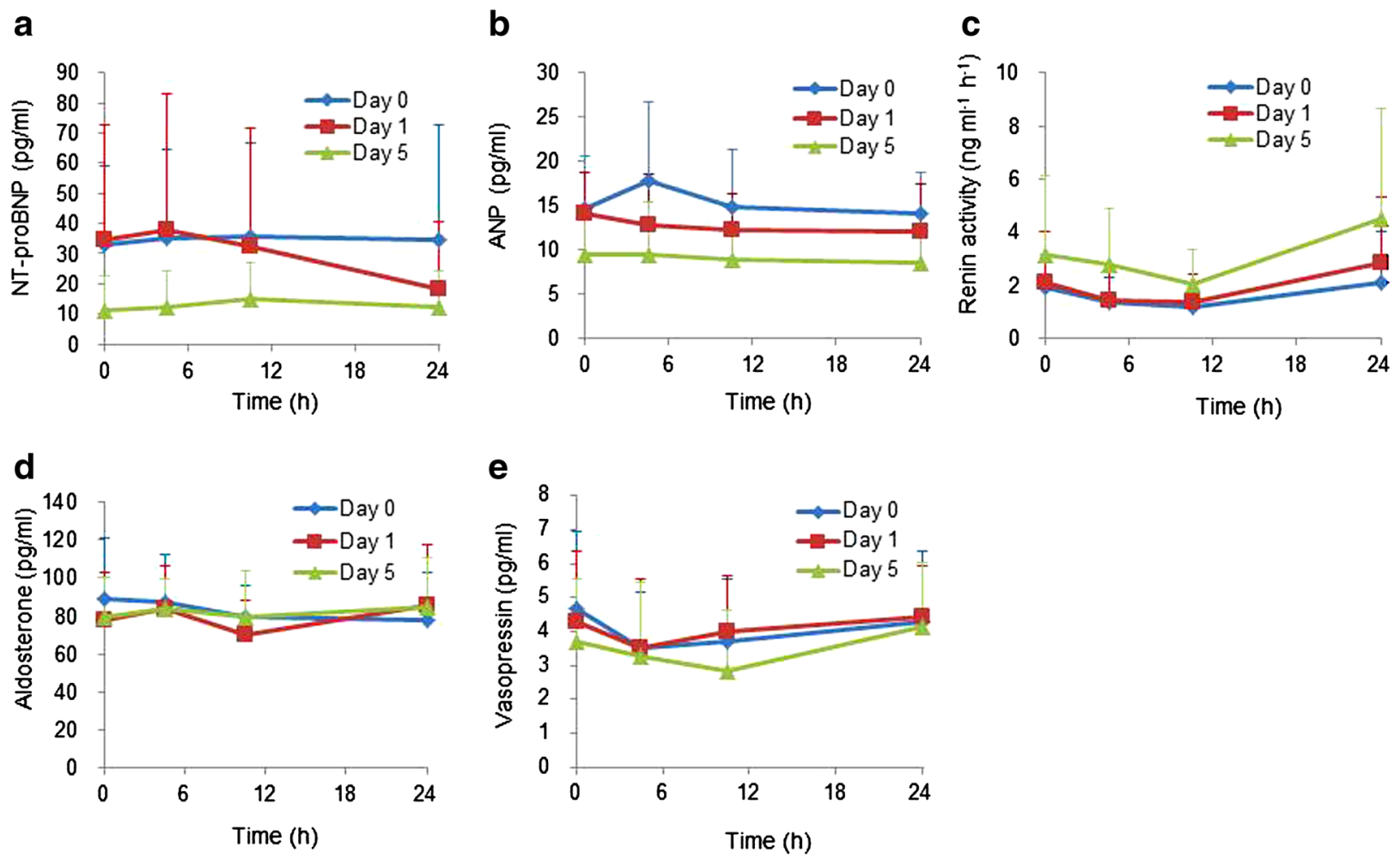

Fig. 2 Canagliflozin-induced changes in hormones levels and enzyme activity involved in homeostasis of body fluid and mineral balance. Changes in a NT-proBNP, b ANP,

c renin activity, $\mathbf{d}$ aldosterone, and e vasopressin. Numbers of patients: day 0 and $1, n=13$; day $5, n=12$. Data are expressed as mean $\pm \mathrm{SD}$

urinary glucose excretion. Actual value of urine volume was correlated with water intake (Days 0 , 1 , and 6), weakly correlated with urinary $\mathrm{Na}$ and urinary K excretions (Days 0, 1, and 6, and Days 1 and 2 , respectively), negatively correlated with urine osmolality (Days 0, 1, and 6), and was not correlated with urine glucose excretion (ESM Table 2). The scatter plot of change in urine volume vs change in each factors, or actual urine volume vs these factors in both studies are shown in ESM Fig. 1.

To identify the factors that are most likely to affect the urine volume change on Day 1, multiple regression analysis (stepwise variable selection method) was performed with change in urine volume as the dependent variable and changes in related factors (water intake, urinary excretion of glucose, $\mathrm{Na}, \mathrm{K}$, plasma NT-proBNP, ANP, renin activity, aldosterone and vasopressin) as independent variables. The change in urinary $\mathrm{Na}$ excretion $(p=0.0006)$ and water intake $(p=0.0239)$ were selected as determinants of urine volume increase $\left(R^{2}=0.7630\right)$ on Day 1 (Table 5). Similar analyses were performed using previous study data [19], and the changes in urinary $\mathrm{Na}$ excretion $(p=0.0031)$ and water consumption $(p<0.0001)$ were selected as determinants of urine volume increase $\left(R^{2}=0.4153 ; \quad\right.$ Table 5$) . \quad$ The regression coefficients of the variables were similar in this and the previous studies: 8.837 and 7.496 for urinary $\mathrm{Na}$ excretion and 0.420 and 0.361 for water intake, respectively.

\section{Effect on Blood Glucose and Total GLP-1}

Figure 3 shows the 24-h blood glucose profile, AUC over $2 \mathrm{~h}$ for postprandial glucose after each 
Table 3 Effect of canagliflozin on AUC $_{0-24 \mathrm{~h}}$ of plasma NT-proBNP, ANP, renin activity, aldosterone, and vasopressin

\begin{tabular}{|c|c|c|c|c|c|c|c|}
\hline & \multirow[t]{2}{*}{ Time point } & \multirow[t]{2}{*}{$n$} & \multicolumn{2}{|c|}{ Actual value } & \multicolumn{3}{|c|}{ Changes from Day 0} \\
\hline & & & Mean & SD & Mean & SD & 95\% CI \\
\hline \multirow[t]{3}{*}{ NT-proBNP (pg mL $\left.{ }^{-1} h\right)$} & Day 0 & 13 & 844.9 & 726.9 & & & \\
\hline & Day 1 & 13 & 716.4 & 841.3 & -128.5 & 451.0 & -401.1 to 144 \\
\hline & Day 5 & 12 & 322.4 & 279.9 & -491.1 & 609.2 & -878.2 to -104 \\
\hline \multirow[t]{3}{*}{ ANP $\left(\mathrm{pg} \mathrm{mL}^{-1} \mathrm{~h}\right)$} & Day 0 & 13 & 365.94 & 144.50 & & & \\
\hline & Day 1 & 13 & 300.13 & 106.71 & -65.81 & 81.49 & -115.1 to -16.6 \\
\hline & Day 5 & 12 & 215.74 & 94.70 & -129.31 & 113.88 & -201.7 to -57 \\
\hline \multirow[t]{3}{*}{ Renin activity $(\mathrm{ng} / \mathrm{mL})$} & Day 0 & 13 & 36.91 & 26.87 & & & \\
\hline & Day 1 & 13 & 45.18 & 33.66 & 8.27 & 16.93 & -1.96 to 18.50 \\
\hline & Day 5 & 12 & 71.80 & 51.52 & 32.47 & 28.36 & 14.45 to 50.49 \\
\hline \multirow[t]{3}{*}{ Aldosterone $\left(\mathrm{pg} \mathrm{mL}^{-1} \mathrm{~h}\right)$} & Day 0 & 13 & 1955.9 & 442.4 & & & \\
\hline & Day 1 & 13 & 1877.7 & 498.9 & -78.2 & 329.8 & -277.5 to 121.1 \\
\hline & Day 5 & 12 & 1971.5 & 468.9 & 37.5 & 350.9 & -185.5 to 260.5 \\
\hline \multirow[t]{3}{*}{ Vasopressin $\left(\mathrm{pg} \mathrm{mL}^{-1} \mathrm{~h}\right)$} & Day 0 & 13 & 94.08 & 44.46 & & & \\
\hline & Day 1 & 13 & 97.12 & 37.02 & 3.03 & 15.64 & -6.41 to 12.48 \\
\hline & Day 5 & 12 & 81.13 & 43.71 & -12.44 & 36.49 & -35.62 to 10.74 \\
\hline
\end{tabular}

$A N P$ atrial natriuretic peptide, $N T$-proBNP $\mathrm{N}$-terminal pro-b-type natriuretic peptide

meal, total GLP-1 concentration, and total GLP-1 AUC over $2 \mathrm{~h}$ after breakfast. Canagliflozin reduced the mean 24-h blood glucose and AUC for postprandial glucose after each meal on Day 1, and the reductions were further enhanced as drug administration continued (Fig. 3a, b). The increases in total blood GLP-1 after breakfast on Days 1 and 5 were higher than those before treatment (Day 0; Fig. 3c, d; ESM Table 3).

\section{Effect on Laboratory Tests}

Plasma insulin was reduced by canagliflozin treatment. Plasma ketone bodies increased on Day 1 and remained elevated. Body weight and blood pressure were not appreciably changed by the treatment (ESM Table 4).
AEs

One female patient withdrew from the study because of anorexia, malaise, nausea, and vomiting. The investigator concluded that the possibility that these AEs were causally related to the study drug could not be excluded, but circumstantial effects and stress such as hospitalization and changes in food may have contributed to their occurrence. The patient quickly recovered from the AEs after discontinuation. No other AEs were reported.

\section{DISCUSSION}

The present study demonstrated that canagliflozin produced a mild increase in urine 
Table 4 Spearman's correlations between urine volume and factors known to affect urine volume in the present study

\begin{tabular}{|c|c|c|c|c|c|}
\hline \multirow[t]{3}{*}{ Factors } & \multicolumn{5}{|c|}{ Urine volume } \\
\hline & \multicolumn{2}{|c|}{ Changes from Day 0} & \multicolumn{3}{|c|}{ Actual values } \\
\hline & $\begin{array}{l}\text { Day } 1 \\
n=13 \\
r\end{array}$ & $\begin{array}{l}\text { Day } 5 \\
n=12 \\
r\end{array}$ & $\begin{array}{l}\text { Day 0 } \\
n=13 \\
r\end{array}$ & $\begin{array}{l}\text { Day } 1 \\
n=13 \\
r\end{array}$ & $\begin{array}{l}\text { Day } 5 \\
n=12 \\
r\end{array}$ \\
\hline Urinary glucose excretion ${ }^{a}$ & $0.764^{*}$ & -0.287 & $0.555^{*}$ & $0.819^{*}$ & 0.287 \\
\hline Water intake ${ }^{a}$ & 0.456 & 0.322 & $0.879^{*}$ & $0.813^{*}$ & $0.827^{*}$ \\
\hline Urinary $\mathrm{Na}$ excretion $^{\mathrm{a}}$ & $0.769^{*}$ & 0.280 & 0.192 & 0.159 & 0.238 \\
\hline Urinary $\mathrm{K}$ excretion $^{\mathrm{a}}$ & $0.725^{*}$ & -0.168 & -0.033 & 0.280 & 0.497 \\
\hline Urine osmolality $^{a}$ & -0.132 & -0.552 & $-0.890^{*}$ & $-0.736^{*}$ & $-0.909^{*}$ \\
\hline Plasma NT-proBNP ${ }^{\mathrm{b}}$ & 0.126 & 0.154 & 0.478 & 0.448 & 0.538 \\
\hline Plasma $\mathrm{ANP}^{\mathrm{b}}$ & 0.374 & 0.385 & 0.346 & 0.104 & 0.559 \\
\hline Plasma renin activity ${ }^{\mathrm{b}}$ & 0.137 & -0.308 & -0.412 & 0.060 & -0.063 \\
\hline Plasma aldosterone $\mathrm{b}^{\mathrm{b}}$ & -0.319 & $-0.706^{*}$ & 0.214 & 0.088 & -0.119 \\
\hline Plasma vasopressin ${ }^{\mathrm{b}}$ & -0.176 & 0.287 & -0.291 & -0.291 & -0.084 \\
\hline
\end{tabular}

$A N P$ atrial natriuretic peptide, $N T$-proBNP $\mathrm{N}$-terminal pro-b-type natriuretic peptide

${ }^{*} p<0.05$

a $24 \mathrm{~h}$

b $\mathrm{AUC}_{0-24 \mathrm{~h}}$

Table 5 Multiple regression analysis of changes in urine volume and factors that affect urine volume on Day 1

\begin{tabular}{|c|c|c|c|c|}
\hline \multirow[b]{2}{*}{ Variable } & \multicolumn{2}{|l|}{$\begin{array}{l}\text { Present study } \\
n=13\end{array}$} & \multicolumn{2}{|l|}{$\begin{array}{l}\text { Previous study } \\
n=39\end{array}$} \\
\hline & Regression coefficient & $p$ & Regression coefficient & $p$ \\
\hline Intercept & -52.707 & 0.6132 & 92.566 & 0.4009 \\
\hline Change in urinary $\mathrm{Na}$ excretion & 8.837 & 0.0006 & 7.496 & $<0.0001$ \\
\hline Change in water intake & 0.420 & 0.0239 & 0.361 & 0.0031 \\
\hline$R^{2}$ & 0.7630 & & 0.4153 & \\
\hline
\end{tabular}

$R^{2}$ coefficient of determination

a The pharmacological effect of canagliflozin on urinary glucose excretion or renal threshold for glucose excretion is nearly saturated at doses above $100 \mathrm{mg}$ [19]. Therefore, we performed a similar analysis using all data with different doses over $100 \mathrm{mg}$ combined in that study to confirm the reproducibility of the present study

volume in patients with T2DM on Day 1. Other changes included increased urinary glucose excretion and urine osmolality on Day 1, which were sustained during the study. A transient increase in urinary $\mathrm{Na}$ excretion on Day 1, decrease in NT-proBNP $\mathrm{AUC}_{0-24 \mathrm{~h}}$ from Day 1 onward, decrease in ANP $\mathrm{AUC}_{0-24 \mathrm{~h}}$ and increase in renin activity $\mathrm{AUC}_{0-24 \mathrm{~h}}$ on Day 5, 

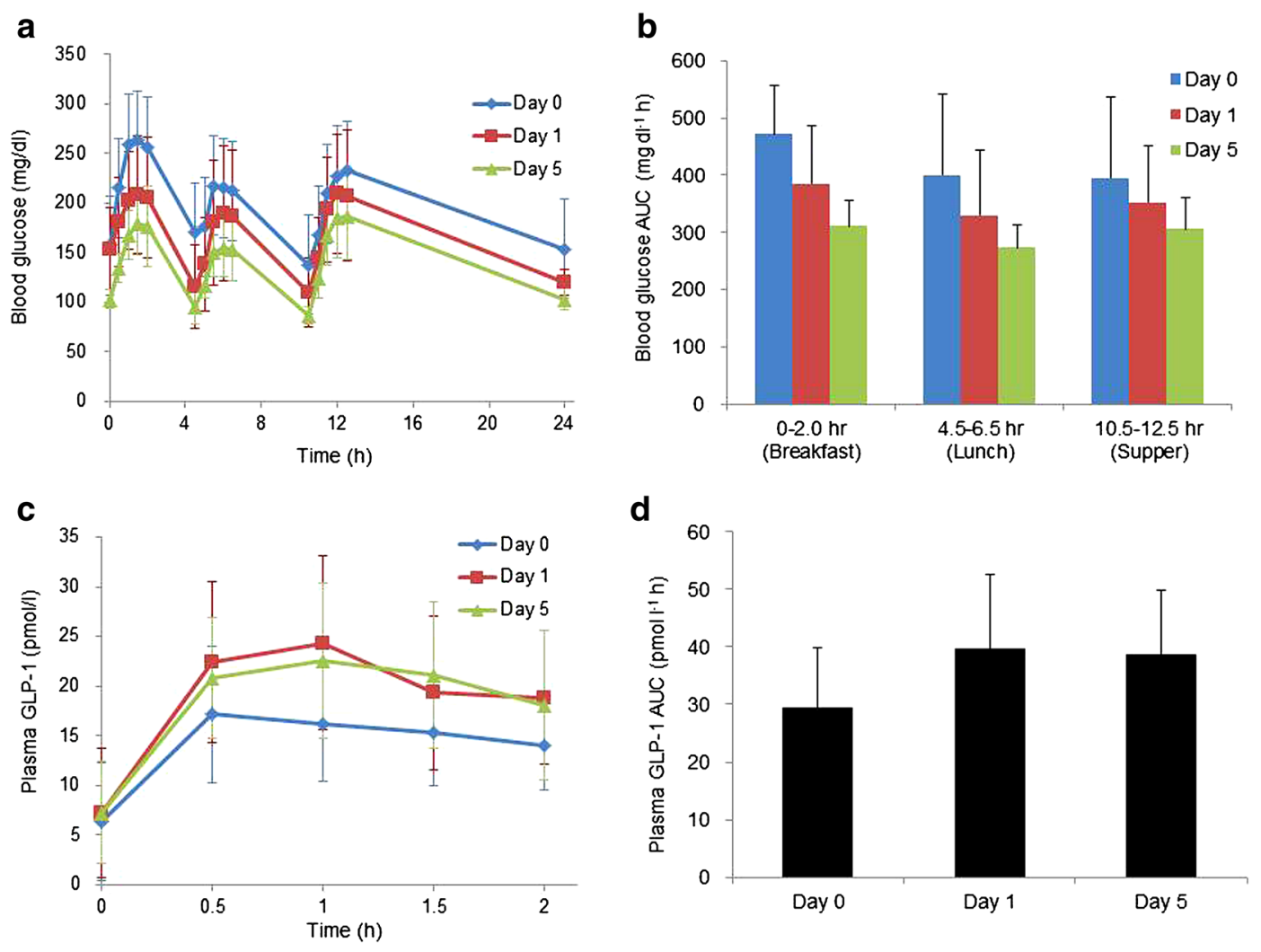

Fig. 3 Canagliflozin-induced changes in blood glucose and total GLP-1 after meals. Changes in a plasma glucose concentration over time, $\mathbf{b}$ postprandial blood glucose AUC after each meal, c plasma total GLP-1 after breakfast,

and $\mathbf{d}$ total GLP-1 AUC $_{0-2 \mathrm{~h}}$ after breakfast. Numbers of patients: day 0 and $1, n=13$; day $5, n=12$. Data are expressed as mean $\pm \mathrm{SD}$

but no appreciable changes in plasma vasopressin and aldosterone, were observed. We performed correlation and multiple regression analyses using the data of present and previous studies [19]. Regarding changes from baseline, $\mathrm{Na}$, and $\mathrm{K}$ were correlated with urine volume. Meanwhile, the actual value of urine volume was correlated with water intake on Days 0, 1, and 5. Multiple regression analysis showed changes in $\mathrm{Na}$ excretion and water intake as factors that affected urine volume change on Day 1. Interestingly, two same factors were selected and the regression coefficients were closed in both studies, which suggested the reproducibility of the present study. Water intake was highly correlated with

actual urine volume before and during treatment, and was not affected by the treatment; the correlation is thought to reflect the general body response. Meanwhile, canagliflozin induced transient $\mathrm{Na}$ excretion on Day 1 , and the change in $\mathrm{Na}$ excretion was closely correlated with change in urine volume. Thus, the transient increase in $\mathrm{Na}$ excretion is thought to have played a major role in the canagliflozin-induced increase in urine volume.

In the present study, change in urinary glucose excretion was correlated with change in urine volume on Day 1. It should be noted that urinary glucose excretion was fairly constant from Day 1 to Day 5, despite urine volume, as well as urinary $\mathrm{Na}$ excretion, 
returning to baseline from Day 2 to Day 5. In addition, there is no correlation between urine volume and urinary glucose excretion in the previous study. Furthermore, urinary glucose excretion was not selected by multiple regression analysis. Collectively, canagliflozin-induced diuresis is likely to reflect natriuresis, not glucose-driven osmotic diuresis. This is consistent with the results of a recent simulation study using a mathematical model of the rat nephron, which demonstrated that SGLT2 inhibition-induced increase in urine volume is expected to reflect natriuresis rather than osmotic diuresis [23]. Increases in urine output and urinary $\mathrm{Na}$ excretion were also reported in Japanese patients with T2DM and heart failure after treatment with ipragliflozin for 4 days [24], although the patient profile was different from that in the present study.

In the present and previous studies, the actual value of urine osmolality was negatively correlated with the actual value of urine volume before and during treatment. In general, increasing urine volume dilutes the solutes, resulting in decreased urine osmolality. The negative correlation between increased urine volume and urine osmolality is also likely to be attributed to the physiological response.

The present study also demonstrated that canagliflozin decreased plasma ANP and NT-proBNP levels and increased plasma renin activity. Change in plasma aldosterone was negatively correlated with change in urine volume on Day 5. As these factors are involved in regulating $\mathrm{Na}$ excretion, working in concert or counteracting each other in the distal portion of the nephron, these changes may contribute to increased renal tubular reabsorption of $\mathrm{Na}$ in a compensatory manner. A similar compensatory mechanism was reported for the action of diuretics such as furosemide and hydrochlorothiazide, with the natriuretic action abolished by continued treatment [25], which is considered to be mediated through a decrease in plasma ANP and activation of the renin-angiotensin system.

The 4-day ipragliflozin treatment significantly decreased plasma ANP and BNP and increased plasma renin activity, while plasma aldosterone tended to increase, but without statistical significance [24]. A 12-week treatment with dapagliflozin was reported to be associated with increases in plasma renin activity and aldosterone [26]. The study also demonstrated a slight increase in NT-proBNP by dapagliflozin. The authors offered a possible explanation for these discordant results through a transient rise in erythropoietin, which is known to enhance BNP secretion [27]. Interestingly, in the present study, NT-proBNP was decreased, which generally indicates decreased body fluids, and $\mathrm{Ht}$, BUN/CRE, serum osmolality, or vasopressin did not change appreciably during the study. Furthermore, plasma aldosterone did not increase. Accordingly, the effects of SGLT2 inhibitors on plasma aldosterone have been inconsistent. Furthermore, change in plasma aldosterone was negatively correlated with change in urine output on Day 5 , and thus the physiological role of this relationship remains unclear.

Treatment with canagliflozin induced glycosuria, decreased blood glucose throughout the day, and suppressed postprandial hyperglycemia, with the largest effect after breakfast. In addition, oral administration of $100 \mathrm{mg}$ of canagliflozin enhanced the increase in plasma total GLP-1 after breakfast. Although similar results with higher doses were reported in previous studies on non-Japanese healthy subjects $(300 \mathrm{mg}$ ) and Japanese healthy subjects (200 mg) [28, 29], this is the first report to show that a lower dose of 
canagliflozin(100 mg) increased the total GLP-1 in T2DM patients. As canagliflozin has modest potency for SGLT1 inhibition relative to that of other highly-selective SGLT2 inhibitors [30], and intestinal SGLT1 inhibition enhanced GLP-1 secretion in a rodent model [31], the increase in total GLP-1 may have arisen through inhibition of SGLT1 in the intestine.

The magnitude of the increase in serum ketone bodies in the present study was larger than that in a previous study [32]. This may be attributed to the fact that ketone body levels were elevated immediately after hospitalization (the average of ketone body levels increased from $123.37 \mu \mathrm{mol} / \mathrm{L}$ at screening to $222.29 \mu \mathrm{mol} / \mathrm{L}$ before dosing), possibly through the dietary restriction.

The limitations of the study were the small sample size, short-term nature, and lack of control group. Urine volume is variable among patients, days, and ambient conditions. However, the samples in the present study were obtained from hospitalized patients to ensure the controlled and reliable baseline data (before administration). In addition, we performed similar analyses using our previous study data to evaluate the reproducibility of the present study. Therefore, we think this paper has clinical value. The long-term effect and mechanism of canagliflozin for urine volume remains to be addressed.

\section{CONCLUSIONS}

Natriuresis is suggested to be the primary factor involved in the canagliflozin-induced transient increase in urine output on Day 1. Decreased plasma ANP and NT-proBNP and increased plasma renin activity may be a compensatory mechanism for subsequent urine output recovery.

\section{ACKNOWLEDGEMENTS}

Sponsorship, article processing charges, and the open access charge for this study were funded by Mitsubishi Tanabe Pharma Corporation. The authors would like to acknowledge the assistance of Ms. M. Goda (Science Communication Department, Mitsubishi Tanabe Pharma Corporation), and Dr. M. Katoh (Ikuyaku Medical Research Department, Mitsubishi Tanabe Pharma Corporation) during interpretation of the study data. The authors thank the ASCA Corporation and Dr. A. Saito (International Medical Translation Service, Inc.) for providing editorial support, which was funded by Mitsubishi Tanabe Pharma Corporation. H.T. contributed to the organization of the study and interpretation of data. K.T. contributed to the acquisition of data. H.I., H.K., N.M., T.H., and K.A. contributed to the study design and interpretation of data. M.T. contributed to the conception of the study. N.I. contributed to interpretation of data. K.K. supervised the study, and contributed to the study design and interpretation of data. All named authors meet the International Committee of Medical Journal Editors (ICMJE) criteria for authorship for this manuscript, take responsibility for the integrity of the work as a whole, and have given final approval for the version to be published. All authors had full access to all of the data in this study and take complete responsibility for the integrity of the data and accuracy of the data analysis.

Disclosures. Nobuya Inagaki has received research support from Eli Lilly Japan. K.K., Mitsubishi Tanabe Pharma Corp., and MSD K.K.; received grant from Astellas Pharma Inc., AstraZeneca K.K., Bristol-Myers Squibb Co., 
Chugai Pharmaceutical Co. Ltd., Daiichi Sankyo Co., Ltd., Japan Diabetes Foundation, Japan Tobacco Inc., Kissei Pharmaceutical Co., Ltd., Kyowa Hakko Kirin Co., Ltd., Mitsubishi Tanabe Pharma Corp., MSD K.K., Nippon Boehringer Ingelheim Co., Ltd., Novartis Pharma K.K., Novo Nordisk Pharma Ltd., Ono Pharmaceutical Co., Ltd., Pfizer Japan Inc., Ltd., Sanofi K.K., Sanwa Kagaku Kenkyusho Co., Sumitomo Dainippon Pharma Co., Ltd., Taisho Toyama Pharmaceutical Co. Ltd., and Takeda Pharmaceutical Co., Ltd.,. has received speakers bureaus or consulting fee from Astellas Pharma Inc., MSD K.K.,Nippon Boehringer Ingelheim Co., Ltd., Sanofi K.K., Sumitomo Dainippon Pharma Co., Ltd., and Takeda Pharmaceutical Co., Ltd.

Kohei Kaku has received grant from Nippon Boehringer Ingelheim Co. Ltd., Daiichi Sankyo Co. Ltd., and Mitsubishi Tanabe Pharma Corp.; has received speakers' bureau from Astellas Pharma Inc., AstraZeneca K.K., Fuji Film Pharma Co. Ltd., Kissei Pharmaceutical Co. Ltd., Kowa Company Ltd., Mitsubishi Tanabe Pharma Corp., MSD K.K., Nippon Boehringer Ingelheim Co. Ltd., Novartis Pharma K.K., Novo Nordisk Pharma Ltd., Ono Pharmaceutical Co. Ltd., Sanofi K.K., Sumitomo Dainippon Pharma Co. Ltd., Takeda Pharmaceutical Co. Ltd., and Taisho Toyama Pharmaceutical Co. Ltd. Kazuhiko Takano has nothing to disclose. Hiroyuki Tanaka is an employee of Mitsubishi Tanabe Pharma Corp.Hiroaki Iijima is an employee of Mitsubishi Tanabe Pharma Corp.Hajime Kubo is an employee of Mitsubishi Tanabe Pharma Corp.Nobuko Maruyama is an employee of Mitsubishi Tanabe Pharma Corp.Toshio Hashimoto is an employee of Mitsubishi Tanabe Pharma Corp.Kenji Arakawa is an employee of Mitsubishi Tanabe Pharma Corp.Masanori Togo is an employee of Mitsubishi Tanabe Pharma Corp.
Compliance with Ethical Standards. The study was conducted in accordance with ethical principles that complied with the Declaration of Helsinki (revised October 2013), ethical guidelines for medical and health research involving human subjects, and conflict of interest management rules for clinical studies at the Medical Corporation Hokubukai Utsukushigaoka Hospital (Sapporo, Japan). The study protocol was approved by the Institutional Review Board at the Utsukushigaoka Hospital. All participants provided written informed consent before taking part in the study

Data Availability. The datasets generated during and/or analyzed during the current study are not publicly available because of lack of agreement for disclosing individual raw data in public but are available from the corresponding author on reasonable request.

Open Access. This article is distributed under the terms of the Creative Commons Attribution-NonCommercial 4.0 International License (http://creativecommons.org/licenses/ by-nc/4.0/), which permits any noncommercial use, distribution, and reproduction in any medium, provided you give appropriate credit to the original author(s) and the source, provide a link to the Creative Commons license, and indicate if changes were made.

\section{REFERENCES}

1. Kahn SE, Cooper ME, Del Prato S. Pathophysiology and treatment of type 2 diabetes: perspectives on the past, present, and future. Lancet. 2014;383(9922):1068-83.

2. Gerich JE. Role of the kidney in normal glucose homeostasis and in the hyperglycaemia of diabetes mellitus: therapeutic implications. Diabet Med. 2010;27(2):136-42. 
3. DeFronzo RA, Davidson JA, Del Prato S. The role of the kidneys in glucose homeostasis: a new path towards normalizing glycaemia. Diabetes Obes Metab. 2012;14(1):5-14.

4. Ferrannini E, Solini A. SGLT2 inhibition in diabetes mellitus: rationale and clinical prospects. Nat Rev Endocrinol. 2012;8(8):495-502.

5. Mudaliar S, Polidori D, Zambrowicz B, Henry RR. Sodium-glucose cotransporter inhibitors: effects on renal and intestinal glucose transport: from bench to bedside. Diabetes Care. 2015;38(12):2344-53.

6. Lorber D. Importance of cardiovascular disease risk management in patients with type 2 diabetes mellitus. Diabetes, Metab Syndr Obes. 2014;7:169-83.

7. Scheen AJ. Pharmacodynamics, efficacy and safety of sodium-glucose co-transporter type 2 (SGLT2) inhibitors for the treatment of type 2 diabetes mellitus. Drugs. 2015;75(1):33-59.

8. Fujita $\mathrm{Y}$, Inagaki N. Renal sodium glucose cotransporter 2 inhibitors as a novel therapeutic approach to treatment of type 2 diabetes: clinical data and mechanism of action. J Diabetes Investig. 2014;5(3):265-75.

9. Shyangdan DS, Uthman OA, Waugh N. SGLT-2 receptor inhibitors for treating patients with type 2 diabetes mellitus: a systematic review and network meta-analysis. BMJ Open. 2016;6(2):e009417.

10. Ghosh RK, Bandyopadhyay D, Hajra A, Biswas M, Gupta A. Cardiovascular outcomes of sodium-glucose cotransporter 2 inhibitors: a comprehensive review of clinical and preclinical studies. Int J Cardiol. 2016;1(212):29-36.

11. Zinman B, Wanner C, Lachin JM, Fitchett D, Bluhmki E, Hantel $S$, et al. Empagliflozin, cardiovascular outcomes, and mortality in type 2 diabetes. N Engl J Med. 2015;373(22):2117-28.

12. Fitchett D, Zinman B, Wanner C, Lachin JM, Hantel S, Salsali A, et al. Heart failure outcomes with empagliflozin in patients with type 2 diabetes at high cardiovascular risk: results of the EMPA-REG OUTCOME(R) trial. Eur Heart J. 2016;37(19):1526-34.

13. Perseghin G, Solini A. The EMPA-REG outcome study: critical appraisal and potential clinical implications. Cardiovasc Diabetol. 2016;15(1):85.

14. Lam KS, Chow CC, Tan KC, Ma RC, Kong AP, Tong $\mathrm{PC}$, et al. Practical considerations for the use of sodium-glucose co-transporter type 2 inhibitors in treating hyperglycemia in type 2 diabetes. Curr Med Res Opin. 2016;32(6):1097-108.

15. Sinclair AJ, Bode B, Harris S, Vijapurkar U, Shaw W, Desai M, et al. Efficacy and safety of canagliflozin in individuals aged 75 and older with type 2 diabetes mellitus: a pooled analysis. J Am Geriatr Soc. 2016;64(3):543-52.

16. Plosker GL. Canagliflozin: a review of its use in patients with type 2 diabetes mellitus. Drugs. 2014;74(7):807-24.

17. Rosenthal N, Meininger G, Ways K, Polidori D, Desai M, Qiu R, et al. Canagliflozin: a sodium glucose co-transporter 2 inhibitor for the treatment of type 2 diabetes mellitus. Ann N Y Acad Sci. 2015;1358:28-43.

18. Seufert J. SGLT2 inhibitors-an insulin-independent therapeutic approach for treatment of type 2 diabetes: focus on canagliflozin. Diabetes Metab Syndr Obes. 2015;8:543-54.

19. Iijima $\mathrm{H}$, Kifuji $\mathrm{T}$, Maruyama $\mathrm{N}$, Inagaki $\mathrm{N}$. Pharmacokinetics, Pharmacodynamics, and Safety of Canagliflozin in Japanese Patients with Type 2 Diabetes Mellitus. Adv Ther. 2015;32(8):768-82.

20. Sha S, Devineni D, Ghosh A, Polidori D, Hompesch M, Arnolds S, et al. Pharmacodynamic effects of canagliflozin, a sodium glucose co-transporter 2 inhibitor, from a randomized study in patients with type 2 diabetes. PLoS One. 2014;9(9):e110069.

21. Sasaki T, Seino Y, Fukatsu A, Ubukata M, Sakai S, Samukawa Y. Pharmacokinetics, pharmacodynamics, and safety of luseogliflozin in Japanese patients with type 2 diabetes mellitus: a randomized, single-blind, placebo-controlled trial. Adv Ther. 2015;32(4):319-40.

22. Heise T, Seewaldt-Becker E, Macha S, Hantel S, Pinnetti S, Seman L, et al. Safety, tolerability, pharmacokinetics and pharmacodynamics following 4 weeks' treatment with empagliflozin once daily in patients with type 2 diabetes. Diabetes Obes Metab. 2013;15(7):613-21.

23. Weinstein AM. A mathematical model of the rat nephron: glucose transport. Am J Physiol Renal Physiol. 2015;308(10):F1098-118.

24. Takeuchi T, Dohi K, Omori T, Moriwaki K, Sato Y, Nakamori S, et al. Diuretic effects of sodium-glucose cotransporter 2 inhibitor in patients with type 2 diabetes mellitus and heart failure. Int $\mathrm{J}$ Cardiol. 2015;15(201):1-3.

25. Rennke H, Denker B. Renal pathophysiology: the essentials. 4th ed. Philadelphia: Wolters Kluwer/ Lippincott Williams \& Wilkins; 2014. 
26. Lambers Heerspink HJ, de Zeeuw D, Wie L, Leslie B, List J. Dapagliflozin a glucose-regulating drug with diuretic properties in subjects with type 2 diabetes. Diabetes Obes Metab. 2013;15(9):853-62.

27. Piuhola J, Kerkela R, Keenan JI, Hampton MB, Richards AM, Pemberton CJ. Direct cardiac actions of erythropoietin (EPO): effects on cardiac contractility, BNP secretion and ischaemia/ reperfusion injury. Clin Sci (Lond). 2008;114(4):293-304.

28. Polidori D, Sha S, Mudaliar S, Ciaraldi TP, Ghosh A, Vaccaro N, et al. Canagliflozin lowers postprandial glucose and insulin by delaying intestinal glucose absorption in addition to increasing urinary glucose excretion: results of a randomized, placebo-controlled study. Diabetes Care. 2013;36(8):2154-61.

29. Kinoshita S, Kondo K. Evaluation of pharmacokinetic and pharmacodynamic interactions of canagliflozin and teneligliptin in
Japanese healthy male volunteers. Expert Opin Drug Metab Toxicol. 2015;11(1):7-14.

30. Grempler R, Thomas L, Eckhardt M, Himmelsbach F, Sauer A, Sharp DE, et al. Empagliflozin, a novel selective sodium glucose cotransporter-2 (SGLT-2) inhibitor: characterisation and comparison with other SGLT-2 inhibitors. Diabetes Obes Metab. 2012;14(1):83-90.

31. Oguma T, Nakayama K, Kuriyama C, Matsushita Y, Yoshida K, Hikida K, et al. Intestinal sodium glucose cotransporter 1 inhibition enhances glucagon-like peptide- 1 secretion in normal and diabetic rodents. J Pharmacol Exp Ther. 2015;354(3):279-89.

32. Inagaki N, Goda M, Yokota S, Maruyama N, Iijima H. Safety and efficacy of canagliflozin in Japanese patients with type 2 diabetes mellitus: post hoc subgroup analyses according to body mass index in a 52-week open-label study. Expert Opin Pharmacother. 2015;16(11):1577-91. 